PESQUIMAT, Revista de la Fac.CC.MM. de la

UNIVERSIDAD NACIONAL MAYOR DE SAN MARCOS

Vol.III, $N^{\circ} 1$,pag.1-15,LIMA-PERÚ. Julio 2000

\title{
SOBRE EL NÚMERO DE MILNOR
}

Renato Benazic Tomé

e-mail: d230008@unmsm.edu.pe

\section{Universidad Nacional Mayor de San Marcos \\ Facultad de Ciencias Matemáticas}

\begin{abstract}
En el presente trabajo, usando métodos algebraicos, demostramos un resultado que relaciona la multiplicidad algebraica en una singularidad dicrítica aislada de un campo vectorial holomorfo de dimensión compleja tres, con el número de Milnor del campo y el de su transformado estricto.
\end{abstract}

\section{INTRODUCCIÓN}

Sea $\mathcal{M}^{n}$ una variedad analítica compleja de dimensión $n$ y consideremos en ella una foliación analítica singular por curvas. Esto significa que en cualquier punto $p \in \mathcal{M}^{n}$ la.foliación es generada por el campo vectorial holomorfo

$$
Z=\sum_{i=1}^{n} Z_{i} \frac{\partial}{\partial z_{i}}, Z_{1}, Z_{2}, \ldots, Z_{n} \in \mathcal{O}_{n, p} ; \text { y } \quad \text { m.c.d. }\left(Z_{1}, Z_{2}, \ldots, Z_{n}\right)=1
$$

en donde $\mathcal{O}_{n, p}$ es el anillo de gérmenes de las funciones analíticas en $p$. En lo sucesivo, denotaremos por $\mathcal{F}_{Z}$ a esta foliación, diremos que el campo $Z$ genera la foliación $\mathcal{F}_{Z}$ y las funciones $Z_{i}$ serán llamadas componentes de $Z$. El lector interesado en conocer detalles de la teoría de funciones analíticas de varias complejas, deberá consultar [9] y para los que deseen profundizar en la teoría de las variedades analíticas complejas, recomendamos [11].

Sea $p \in \mathcal{M}^{n}$ y consideremos una carta $(U, \phi)$ de $\mathcal{M}^{n}$ alrededor del punto $p$ tal que $\phi(p)=0 \in \mathbb{C}^{n}$, claramente $Z_{i} \circ \phi^{-1}$ es una función analítica de varias variables complejas definida en una vecindad del origen y por lo tanto, ella tiene un desarrollo en series de potencias

$$
Z_{i} \circ \phi^{-1}=\sum_{k \geq 0} Z_{i}^{k}, \quad 1 \leq i \leq n
$$


donde los $Z_{i}^{k}$ son polinomios homogéneos de grado $k$ en $n$ variables complejas. El orden de $Z_{i} \circ \phi^{-1}$ en el $0 \in \mathbb{C}^{n}$ es, por definición, el menor número entero $\nu_{i}$ tal que $Z_{i}^{k} \equiv 0$, para todo $k<\nu_{i}$ y $A_{i}^{\nu_{i}} \not \equiv 0$. No es dificil probar que el número $\nu_{i}$ es independiente de la elección de la carta $(U, \phi)$, por esta razón el entero $\nu_{i}$ es llamado el orden de $Z_{i}$ en $p$ y lo denotamos por $\operatorname{ord}_{p}\left(Z_{i}\right)$. La multiplicidad algebraica de la foliación $\mathcal{F}_{Z}$ (o del campo $Z$ ) en el punto $p \in \mathcal{M}^{n}$, denotada por $m_{p}\left(\mathcal{F}_{Z}\right)$ (o simplemente por $m_{p}(Z)$ ), es definida como el mínimo de los órdenes $\operatorname{ord}_{p}\left(Z_{i}\right)$.

Un punto $p \in \mathcal{M}^{n}$ es llamado punto singular de la foliación $\mathcal{F}_{Z}$ (o del campo $Z$ ) si y sólo si $m_{p}(Z) \geq 1$, en caso contrario decimos que $p$ es un punto regular. El conjunto de todos los puntos singulares de la foliación $\mathcal{F}_{Z}$ será denotado por $\operatorname{Sing}\left(\mathcal{F}_{Z}\right)$. Un punto $p \in \mathcal{M}^{n}$ es llamado singularidad aislada de $Z$ si y sólo si $p \in \operatorname{Sing}\left(\mathcal{F}_{Z}\right)$ y existe una vecindad abierta $U \subseteq \mathcal{M}^{n}$ de $p$ tal que todos los elementos de $U-\{p\}$ son puntos regulares de $Z$.

Denotemos por $I\left[Z_{1}, Z_{2}, \ldots, Z_{n}\right] \subseteq \mathcal{O}_{n, p}$ al ideal generado por las componentes del campo vectorial holomorfo $Z$. El Número de Milnor del campo $Z$ en el punto $p \in \mathcal{M}^{n}$, denotado por $\mu_{p}(Z)$, es definido como

$$
\mu_{p}(Z)=\operatorname{dim}_{\mathbb{C}}\left(\frac{\mathcal{O}_{n, p}}{I\left[Z_{1}, Z_{2}, \ldots, Z_{n}\right]}\right)
$$

Este número es finito si y sólo si $p \in \mathcal{M}^{n}$ es una singularidad aislada de $Z$ y en este caso $\mu_{p}(Z)$ coincide el grado topológico de la función de Gauss inducido por $Z$ el cual es considerado como un campo vectorial real, en una esfera de dimensión real $2 n-1$, suficientemente pequeña centrada en $p$. El objetivo del presente trabajo es estudiar como cambia el número de Milnor $\mu_{p}(Z)$ cuando hacemos explosiones (blow-up) en el punto singular $p$.

\section{EL BLOW-UP CENTRADO EN UN PUNTO}

Sea $\mathcal{M}^{n}$ una variedad analítica compleja de dimensión $n$ y $p \in \mathcal{M}^{n}$, el blow-up (o explosión) del punto $p$ consiste en reemplazar $p$ por el espacio proyectivo complejo $(n-1)$-dimensional $\mathbb{C} P(n-1)$, considerado como el conjunto de direcciones límites en $p$. Esto se consigue de la siguiente manera: denotemos por $\mathbb{P}_{p}\left(\mathcal{M}^{n}\right)$ al espacio proyectivo asociado al plano tangente $T_{p}\left(\mathcal{M}^{n}\right)$ y sea $v \rightarrow[v]$ la aplicación del paso al cociente $T_{p}\left(\mathcal{M}^{n}\right)-\{0\} \rightarrow \mathbb{P}_{p}\left(\mathcal{M}^{n}\right)$. El blow-up del punto $p$ es el conjunto

$$
\tilde{\mathcal{M}}^{n}=\left(\mathcal{M}^{n}-\{p\}\right) \cup \mathbb{P}_{p}\left(\mathcal{M}^{n}\right)
$$


dotado de una estructura de variedad analítica compleja obtenida al reemplazar toda carta de $\mathcal{M}^{n}$ alrededor del punto $p$ :

$$
\phi=\left(z_{1}, \ldots, z_{n}\right): U \rightarrow \mathbb{C}^{n} \text {, tal que } \phi(p)=0,
$$

por las $n$ cartas $\phi_{i},(i=1, \ldots, n)$ definidas del modo siguiente: sea $P_{i}$ el conjunto de las rectas de $T_{p}\left(\mathcal{M}^{n}\right)$ contenidas en el $\operatorname{Ker}\left(d_{p} z_{i}\right)$,

$$
\begin{gathered}
\phi_{i}: U_{i}=\left(U-z_{i}^{-1}(0)\right) \cup\left(\mathbb{P}_{p}\left(\mathcal{M}^{n}\right)-P_{i}\right) \rightarrow \mathbb{C}^{n}, \\
\phi_{i}=\left(\frac{z_{1}}{z_{i}}, \ldots, \frac{z_{i-1}}{z_{i}}, z_{i}, \frac{z_{i+1}}{z_{i}}, \ldots, \frac{z_{n}}{z_{i}}\right) \text { sobre } U \\
\phi_{i}\left(\left[\sum \alpha_{j} \frac{\partial}{\partial z_{j}}\right]\right)=\left(\frac{\alpha_{1}}{\alpha_{i}}, \ldots, \frac{\alpha_{i-1}}{\alpha_{i}}, 0, \frac{\alpha_{i+1}}{\alpha_{i}}, \ldots, \frac{\alpha_{n}}{\alpha_{i}}\right) .
\end{gathered}
$$

En lo sucesivo, la función analítica $E: \tilde{\mathcal{M}}^{n} \rightarrow \mathcal{M}^{n}$ definida por

$$
E(z)= \begin{cases}z, & \text { si } z \notin \mathbb{P}_{p}\left(\mathcal{M}^{n}\right), \\ p, & \text { si } z \in \mathbb{P}_{p}\left(\mathcal{M}^{n}\right) .\end{cases}
$$

será llamada blow-up con centro en el punto $p \in \mathcal{M}^{n}$. La restricción de $E$ a $\tilde{\mathcal{M}}^{n}-\mathbb{P}_{p}\left(\mathcal{M}^{n}\right)$ es un difeomorfismo sobre $\mathcal{M}^{n}-\{p\}$, que en la carta $\left(U_{i}, \phi_{i}\right)$ se escribe como

$$
E\left(z_{1}, \ldots, z_{n}\right)=\left(z_{i} z_{1}, \ldots, z_{i} z_{i-1}, z_{i}, z_{i} z_{i+1}, \ldots, z_{i} z_{n}\right) .
$$

Cuando $\mathcal{M}^{n}=\mathbb{C}^{n}$ y $p=0$, denotamos por $\left(z_{1}, \bar{z}\right)=\left(z_{1}, z_{2}, \ldots, z_{n}\right)$ las coordenadas de $\mathbb{C}^{n}$ y por

$$
t^{\prime}:\left[\left(z_{1}, \bar{z}\right)\right] \rightarrow \frac{\bar{z}}{z_{1}}
$$

la primera carta canónica sobre $U_{1}=\mathbb{C} P(n-1)-\mathbb{P}_{1}$, las otras cartas se obtienen permutando las variables de $\mathbb{C}^{n}$. Sea $\pi: \tilde{\mathbb{C}}^{n} \rightarrow \mathbb{C} P(n-1)$ la fibración de fibra $\mathbb{C}$ definida por

$$
\pi(z)= \begin{cases}{[z],} & \text { si } z \notin \mathbb{C} P(n-1), \\ z, & \text { si } z \in \mathbb{C} P(n-1) .\end{cases}
$$

La carta $\phi_{1}=\left(z_{1}, \bar{z}\right)_{1}$ de $\tilde{\mathbb{C}}^{n}$ definida anteriormente, en este caso se escribe

$$
\left(z_{1} \circ E, t^{\prime} \circ \pi\right): \tilde{U}_{1}=\pi^{-1}\left[U_{1}\right] \rightarrow \mathbb{C}^{n} .
$$

la que designamos, por abuso de notación, como

$$
\left(z_{1}, \bar{z}\right)_{1}=\left(z_{1}, t^{\prime}\right) \quad \text { donde } \quad t^{\prime}=\frac{\bar{z}}{z_{1}}
$$

y en esta carta tenemos: 


$$
E\left(z_{1}, t^{\prime}\right)=\left(z_{1}, z_{1} t^{\prime}\right)
$$

las otras $n-1$ cartas asociadas se obtienen permutando las variables. Por ejemplo, en el caso particular en que $n=3$, se tiene $E(x, t, s)=$ $(x, x t, x s)=\left(z_{1}, z_{2}, z_{3}\right)$ (donde $\left.t^{\prime}=(t, s)\right), E(u, y, v)=(u y, y, v y)=$ $\left(z_{1}, z_{2}, z_{3}\right)$ (donde $\left.t^{\prime}=(u, v)\right)$ y $E(r, w, z)=(r z, w z, z)=\left(z_{1}, z_{2}, z_{3}\right)$ (donde $\left.t^{\prime}=(r, w)\right)$.

Lo desarrollado hasta aquí sobre el blow-up, es suficiente para nuestros propósitos, el lector interesado en mayores detalles sobre el tema, puede consultar las referencias [8], [10] y [5].

\section{TRANSFORMADO ESTRICTO DE FOLIACIONES}

Sea $\mathcal{M}^{n}$ una variedad analítica compleja de dimensión $n$ y consideremos en ella una foliación analítica singular por curvas $\mathcal{F}_{Z}$. Si $E: \tilde{\mathcal{M}}^{n} \rightarrow \mathcal{M}^{n}$ es el blow-up centrado en el punta $p \in \mathcal{M}^{n}$, singularidad aislada de $\mathcal{F}_{Z}$, en la presente sección, vamos a probar que existe una única manera de extender el pull-back $E^{*}\left(\mathcal{F}_{Z}-\{p\}\right)$ a una foliación analítica singular por curvas, denotada por $\tilde{\mathcal{F}}_{Z}$, la cual está definida en una vecindad del espacio proyectivo $\mathbb{C} P(n-1)=E^{-1}(p) \subset \mathcal{M}^{n}$ y cuyo conjunto singular tiene codimensión $\geq 2$. Esta foliación $\tilde{\mathcal{F}}_{Z}$ es llamada la transformada estricta de $\mathcal{F}_{Z}$ por $E$ y el espacio proyectivo $E^{-1}(p)$ es llamado divisor. Denotaremos por $\tilde{Z}$ al campo vectorial holomorfo que genera a la foliación $\tilde{\mathcal{F}}_{Z}$. El punto $p \in \mathcal{M}^{n}$ es llamado singularidad no dicrítica de $\mathcal{F}_{Z}$ si y sólo si el divisor es invariante por $\tilde{\mathcal{F}}_{Z}$, esto significa que $E^{-1}(p)$ es la unión de hojas y singularidades de $\tilde{\mathcal{F}}_{Z}$. En caso contrario, decimos que $p$ es una singularidad dicrítica de $\mathcal{F}_{Z}$. A continuación, detallaremos estos resultados.

Tomemos un sistema de coordenadas locales $z=\left(z_{1}, z_{2}, \ldots, z_{n}\right)$ de una vecindad del punto $p$ tal que $z(p)=0 \in \mathbb{C}^{n}$. En estas coordenadas, la foliación es generada por el campo $Z=\sum_{i=1}^{n} Z_{i} \frac{\partial}{\partial z_{i}}$. Si $m_{0}(Z)=\nu$ (donde $\nu \geq 1$ ), entonces las componentes $Z_{i}$ tiene un desarrollo en serie de potencias alrededor del $0 \in \mathbb{C}^{n}$

$$
Z_{i}=\sum_{k \geq \nu} Z_{k}^{i}, \quad 1 \leq i \leq n,
$$

donde cada $Z_{k}^{i}$ es un polinomio homogéneo de grado $k$.

Consideremos el sistema de coordenadas $\left(y_{1}, y_{2}, \ldots, y_{n}\right)$ de $\tilde{\mathbb{C}}^{n}$ en donde $E$ se expresa como:

$$
E\left(y_{1}, y_{2}, \ldots, y_{n}\right)=\left(y_{1} y_{j}, \ldots, y_{j-1} y_{j}, y_{j+1} y_{j}, \ldots, y_{n} y_{j}\right)=\left(z_{1}, z_{2}, \ldots, z_{n}\right) .
$$


En esta carta, el pull-back de $Z$ por $E$ es dado por

$$
E^{*} Z=Z_{j} \circ E \frac{\partial}{\partial y_{j}}+\sum_{1 \leq i \neq j \leq n}\left(\frac{Z_{i} \circ E-y_{i} Z_{j} \circ E}{y_{j}}\right) \frac{\partial}{\partial y_{i}} .
$$

Pero

$$
\left(Z_{i} \circ E\right)(y)=\sum_{k \geq \nu} y_{j}^{k} Z_{k}^{i}(\bar{y})
$$

en donde $y=\left(y_{1}, \ldots, y_{n}\right)$ e $\bar{y}=\left(y_{1}, \ldots, y_{j-1}, 1, y_{j+1}, \ldots, y_{n}\right)$. Luego:

$$
\begin{aligned}
E^{*} Z(y)= & \left(\sum_{k \geq \nu} y_{j}^{k} Z_{k}^{j}(\bar{y})\right) \frac{\partial}{\partial y_{j}}+ \\
& +\sum_{1 \leq i \neq j \leq n}\left(\sum_{k \geq \nu} y_{j}^{k-1}\left[A_{k}^{i}(\bar{y})-y_{i} A_{k}^{j}(\bar{y})\right]\right) \frac{\partial}{\partial y_{i}}
\end{aligned}
$$

se presentan dos posibilidades:

(i) Existe $i \in\{1,2, \ldots, n\}, i \neq j$ tal que $A_{\nu}^{i}(\bar{y})-y_{i} A_{\nu}^{j}(\bar{y}) \not \equiv 0$. En este caso, $E^{*} Z$ es divisible por $y_{j}^{\nu-1}$ y entonces podemos definir $\tilde{Z}=\frac{E^{*} Z}{y_{j}^{\nu-1}}$. De (3.1) tenemos

$$
\begin{aligned}
\tilde{Z}= & y_{j} Z_{\nu}^{j}(\bar{y}) \frac{\partial}{\partial y_{j}}+\sum_{1 \leq i \neq j \leq n}\left(A_{\nu}^{i}(\bar{y})-y_{i} A_{\nu}^{j}(\bar{y})\right) \frac{\partial}{\partial y_{i}}+ \\
& +y_{j} \tilde{Y}(y)
\end{aligned}
$$

en donde $\tilde{Y}$ es un campo vectorial holomorfo. Denotaremos por $\tilde{\mathcal{F}}_{Z}$ a la foliación generada por $\tilde{Z}$. De (3.2) se deduce fácilmente que el divisor $E^{-1}(0)$ (el cual, en la carta que estamos trabajando, se expresa como $\left.E^{-1}(0)=\left\{\left(y_{1}, y_{2}, \ldots, y_{n}\right) \in \mathbb{C}^{n}: y_{j}=0\right\}\right)$, es invariante por la foliación $\tilde{\mathcal{F}}_{Z}$. En este caso decimos que $0 \in \mathbb{C}^{n}$ es una Singularidad no dicrítica de $Z$.

(ii) $A_{\nu}^{i}(\bar{y})-y_{i} A_{\nu}^{j}(\bar{y}) \equiv 0$, para todo $1 \leq i \leq n, i \neq j$. En este caso, $E^{*} Z$ es divisible por $y_{j}^{\nu}$ y entonces podemos definir $\tilde{Z}=\frac{E^{*} Z}{y_{j}^{\nu}}$. De (1) tenemos

$$
\tilde{Z}=Z_{\nu}^{j}(\bar{y}) \frac{\partial}{\partial y_{j}}+y_{j} \tilde{Y}(y)
$$


De (3.3) deducimos que $E^{-1}(0)$ no es invariante por $\tilde{\mathcal{F}}_{Z}$, en efecto, ahora las hojas de $\tilde{\mathcal{F}}_{Z}$ son tranversales o tangentes al divisor y el conjunto singular está contenido en $\left\{\bar{y} \in E^{-1}(0): Z_{\nu}^{j}(\bar{y})=0\right\}$. Cuando esto ocurre, decimos que $0 \in \mathbb{C}^{n}$ es una Singularidad dicrítica de $Z$.

Note que en cualquiera de los dos casos, $E^{*} Z$ y $\tilde{Z}$ coinciden fuera del divisor. La foliación $\tilde{\mathcal{F}}_{Z}$ es llamada transformada estricta de $\mathcal{F}_{Z}$ por E.

\section{EL NÚMERO DE MILNOR DE UN CAMPO VECTORIAL HOLOMORFO}

Sea $U \subseteq \mathbb{C}^{n}$ un abierto, denotemos por $\mathcal{O}_{n, p}$ al anillo de gérmenes de las funciones analíticas en $p \in U$ y sea $I\left[Z_{1}, Z_{2}, \ldots, Z_{n}\right] \subseteq \mathcal{O}_{n, p}$ el ideal generado por las componentes del campo vectorial holomorfo $Z$ definido en $U$. El Número de Milnor del campo $Z$ en el punto $p$, denotado por $\mu_{p}(Z)$, es definido como

$$
\mu_{p}(Z)=\operatorname{dim}_{\mathbb{C}}\left(\frac{\mathcal{O}_{n, p}}{I\left[Z_{1}, Z_{\left.2, \ldots, Z_{n}\right]} \ldots\right.}\right)
$$

Este número satisface las siguientes propiedades (ver [18]):

1. $\mu_{p}(Z)$ es finito si y sólo si $p$ es una singularidad aislada de $Z$.

2. $\mu_{p}(Z)=0$ si y sólo si $p$ es un punto regutar de $Z$.

3. $\mu_{p}(Z)=1$ si y sólo si $\operatorname{det}\left(\frac{\partial Z_{i}(p)}{\partial z_{j}}\right)_{1 \leq i, j \leq n} \neq 0$.

Sean $p \in U$ una singularidad aislada del campo vectorial $Z$, tal que $\mu_{p}(Z)=\nu, \mathcal{F}_{Z}$ la foliación generada por $Z, \tilde{\mathcal{F}}_{Z}$ el transformado estricto de $\mathcal{F}_{Z}$ y $\tilde{Z}$ el campo vectorial holomorfo que genera a la foliación $\tilde{\mathcal{F}}_{Z}$. Cuando $n=2$, existe una fórmula que relaciona $\nu$ con el número de Milnor de $Z$ en $p$ y el número de Milnor de las singularidades de $\tilde{Z}$ (ver $[10])$ :

$\mu_{p}(Z)=\left\{\begin{array}{l}\nu^{2}-\nu-1+\sum_{q \in E^{-1}(p)} \mu_{q}(\tilde{Z}), \text { si } p \text { es una sing. no dicrítica. } \\ \nu^{2}+\nu-1+\sum_{q \in E^{-1}(p)} \mu_{q}(\tilde{Z}), \text { si } p \text { es una sing. dicrítica. }\end{array}\right.$

Observe que como el conjunto $\operatorname{Sing}\left(\tilde{\mathcal{F}}_{Z}\right)$ es finito, las sumatorias que aparecen en la expresión anterior son finitas. Existe una generalización $n$-dimensional de la fórmula anterior bajo las hipótesis de que $p$ es una singularidad aislada no dicrítica de $Z$ y el conjunto $\operatorname{Sing}\left(\tilde{\mathcal{F}}_{Z}\right)$ 
es finito (ver [4]):

$$
\mu_{p}(Z)=\nu^{n}-\nu^{n-1}-\ldots-\nu-1+\sum_{q \in E^{-1}(p)} \mu_{q}(\tilde{Z})
$$

En el presente trabajo, demostraremos un resultado análogo al anterior para el caso en que $p$ es una singularidad aislada dicrítica de un campo vectorial holomorfo $Z$, definido en un abierto de $\mathbb{C}^{3}$. La prueba que presentaremos es algebraica y utiliza algunas herramientas de la Teoría de Intersección de variedades analíticas complejas, las que pasaremos a detallar en la siguiente sección.

\section{ELEMENTOS DE TEORÍA DE INTERSECIÓN}

Denotemos por $\mathcal{O}_{n}$ al anillo de gérmenes de las funciones analíticas en $0 \in \mathbb{C}^{n}$, luego cualquier $F \in \mathcal{O}_{n}$ tiene un desarrollo en series de potencias, definido en una vecindad del origen:

$$
F(z)=\sum_{k \geq 0} F_{k}(z)
$$

en donde los $F_{k}(z)$ son polinomios homogéneos de grado $k \geq 0$. En estas condiciones, el orden de $F$ en 0 , denotado por $\operatorname{ord}_{0}(F)$, es definido como el menor número entero no negativo $m$ tal que $F_{k} \equiv 0$ para todo $k<m$ y $F_{m} \not \equiv 0$.

Es bien conocido que el subconjunto de $\mathbb{C}^{n}$ formado por todos los ceros de $F$, al que denotaremos por $(F=0)$, i.e.

$$
(F=0)=\left\{z \in \mathbb{C}^{n}: F(z)=0\right\}
$$

es una hipersuperficie analítica de $\mathbb{C}^{n}$, es decir, una subvariedad analítica de $\mathbb{C}^{n}$ de codimensión 1 (o de dimensión $n-1$ ). Por ejemplo si $n=2$, $(F=0)$ es una curva analítica en $\mathbb{C}^{2}$ mientras que si $n=3,(F=0)$ es una superficie analítica en $\mathbb{C}^{3}$. En el caso particular de que $F$ es un polinomio, el conjunto $(F=0)$ es una hipersuperficie algebraica de $\mathbb{C}^{n}$.

Sean $F_{1}, F_{2}, \ldots, F_{n} \in \mathcal{O}_{n}$ y denotemos $A_{j}=\left(F_{j}=0\right),(1 \leq j \leq n)$. Vamos a suponer que $\bigcap_{j=1}^{n} A_{j}$ es un conjunto de dimensión cero (conjunto discreto de $\mathbb{C}^{n}$ ). El producto cartesiano $\Pi A_{j}=A_{1} \times A_{2} \times \ldots \times A_{n}$ en $\mathbb{C}^{n^{2}}=\mathbb{C}^{n} \times \mathbb{C}^{n} \times \ldots \times \mathbb{C}^{n}$ tiene dimensión $n(n-1)$, y la diagonal $\Delta=\left\{\left(z_{1}, z_{2}, \ldots, z_{n^{2}}\right) \in \mathbb{C}^{n^{2}}: z_{1}=z_{2}=\ldots=z_{n^{2}}\right\}$ tiene dimensión complementaria $n$. Si $a \in \bigcap_{j=1}^{n} A_{j}$ entonces $(a)^{n}=(a, a, \ldots, a) \in \mathbb{C}^{n^{2}}$ es un punto aislado de $\left(\Pi A_{j}\right) \cap \Delta$, se sigue que la proyección $\left.\pi_{\Delta}\right|_{\Pi A_{j}}$ 
de $\Delta$ sobre $\Delta^{\perp}$ (en $\mathbb{C}^{n^{2}}$ ) es un recubrimiento analítico de alguna vecindad de $(a)^{n}$. El índice de intersección de las hipersuperficies analíticas $A_{1}, A_{2}, \ldots, A_{n}$ en el punto $a \in \bigcap_{j=1}^{n} A_{j}$, denotado por $i_{a}\left(F_{1}, F_{2}, \ldots, F_{n}\right)$, es definido como la multiplicidad (ver [6]) de la proyección $\left.\pi_{\Delta}\right|_{\Pi A_{j}}$ en $(a)^{n} \in \mathbb{C}^{n^{2}}$ :

$$
i_{a}\left(F_{1}, F_{2}, \ldots, F_{n}\right)=\mu_{(a)^{n}}\left(\left.\pi_{\Delta}\right|_{\Pi A_{j}}\right)
$$

Si $a \notin \bigcap_{j=1}^{n} A_{j}$, es conveniente definir $i_{a}\left(F_{1}, F_{2}, \ldots, F_{n}\right)=0$.

Como consecuencia directa de la definición, tenemos que el índice de intersección es una función simétrica de las funciones $F_{1}, F_{2}, \ldots, F_{n}$, i.e.

$$
i_{a}\left(F_{j_{1}}, F_{j_{2}}, \ldots, F_{j_{n}}\right)=i_{a}\left(F_{1}, F_{2}, \ldots, F_{n}\right),
$$

para cualquier permutación $(1,2, \ldots, n) \rightarrow\left(j_{1}, \dot{j}_{2}, \ldots, j_{n}\right)$.

Otras propiedades fundamentales del índice de intersección son las siguientes:

1. $i_{a}\left(F_{1} \cdot F_{1}^{\prime}, F_{2}, \ldots, F_{n}\right)=i_{a}\left(F_{1}, F_{2}, \ldots, F_{n}\right)+i_{a}\left(F_{1}^{\prime}, F_{2}, \ldots, F_{n}\right)$

2. Si $A$ es una matriz cuadrada $n \times n$ invertible, cuyos elementos son funciones holomorfas en una vecindad de $a \in \mathbb{C}^{n}$ y si denotamos:

$$
\left(\begin{array}{c}
F_{1}^{\prime} \\
F_{2}^{\prime} \\
\vdots \\
F_{n}^{\prime}
\end{array}\right)=A\left(\begin{array}{c}
F_{1} \\
F_{2} \\
\vdots \\
F_{n}
\end{array}\right)
$$

entonces $i_{a}\left(F_{1}^{\prime}, F_{2}^{\prime}, \ldots, F_{n}^{\prime}\right)=i_{a}\left(F_{1}, F_{2}, \ldots, F_{n}\right)$

El lector puede encontrar la demostración de estas y otras propiedades del índice de intersección en [6] y [7].

La interpretación geométrica del índice de intersección es la siguiente: Dado $\epsilon>0$ suficientemente pequeño, las $\epsilon$-perturbaciones $A_{j}(\epsilon)=$ $\left(F_{j}=\epsilon\right)$ de $A_{j}$ se interceptan en exactamente $i_{a}\left(F_{1}, F_{2}, \ldots, F_{n}\right)$ puntos, i.e.

$$
\operatorname{card}\left(\bigcap_{j=1}^{n} A_{j}(\epsilon)\right)=i_{a}\left(F_{1}, F_{2}, \ldots, F_{n}\right) .
$$

En estas condiciones, el número de Milnor del campo vectorial holomorfo $Z=\sum_{i=1}^{n} Z_{i} \frac{\partial}{\partial z_{i}}$, puede ser interpretado geométricamente como el 
índice de intersección $i_{p}\left(Z_{1}, Z_{2}, \ldots, Z_{n}\right)$ en $p$ de las $n$ hipersuperficies analíticas generadas por las componentes de $Z$ (ver [6]):

$$
\mu_{p}(Z)=i_{p}\left(Z_{1}, Z_{2}, \ldots, Z_{n}\right)
$$

Sea $E$ el blow-up centrado en 0 , en la carta $\tilde{U}_{j}(1 \leq j \leq n)$ de $\tilde{\mathbb{C}}^{n}$ introducimos las coordenadas $\left(y_{1}, y_{2}, \ldots, y_{n}\right)$. De esta manera $E$ : $\tilde{U}_{j} \rightarrow \mathbb{C}^{n}$ tiene la siguiente expresión:

$E\left(y_{1}, y_{2}, \ldots, y_{n}\right)=\left(y_{1} y_{j}, \ldots, y_{j-1} y_{j}, y_{j+1} y_{j}, \ldots, y_{n} y_{j}\right)=\left(z_{1}, \ldots, z_{n}\right)$

Dado $F \in \mathcal{O}_{n}$, con $\operatorname{ord}_{0}(F)=m$, en la carta $\tilde{U}_{j}$ definimos el transformado estricto de $F$ por $E$, denotado por $\tilde{F}$, como:

$$
\tilde{F}\left(y_{1}, y_{2}, \ldots, y_{n}\right)=\frac{(F \circ E)\left(y_{1}, y_{2}, \ldots, y_{n}\right)}{y_{j}^{m}} .
$$

El Teorema siguiente, cuya demostración-puede ser encontrada en [7], es fundamental para demostrar el resultado principal del presente trabajo:

Teorema 1. Sean $F_{1}, F_{2}, \ldots, F_{n} \in \mathcal{O}_{n}$ tales que:

1. $0 \in \mathbb{C}^{n}$ es un punto de intersección aislado de las hipersuperficies analíticas $\left(F_{1}=0\right),\left(F_{2}=0\right), \ldots,\left(F_{n}=0\right)$.

2. Las hipersuperficies $\left(\tilde{F}_{1}=0\right),\left(\tilde{F}_{2}=0\right), \ldots,\left(\tilde{F}_{n}=0\right)$ tienen puntos de intersecciones aislados en el divisor $E^{-1}(0)$.

Entonces

$$
\begin{aligned}
i_{0}\left(F_{1}, F_{2}, \ldots, F_{n}\right)= & \operatorname{ord}_{0}\left(F_{1}\right) \operatorname{ord}_{0}\left(F_{2}\right) \ldots \operatorname{ord}_{0}\left(F_{n}\right)+ \\
& +\sum_{q \in E^{-1}(p)} i_{q}\left(\tilde{F}_{1}, \tilde{F}_{2}, \ldots, \tilde{F}_{n}\right)
\end{aligned}
$$

\section{EL RESULTADO PRINCIPAL}

Sea $\mathcal{M}^{3}$ una variedad analítica compleja de dimensión 3 y consideremos en ella una follíación analítica singular por curvas. En $p \in \mathcal{M}^{3}$ singularidad aislada dicrítica de la foliación, consideremos un sistema de coordenadas locales $\left(z_{1}, z_{2}, z_{3}\right)$ tal que $p=0 \in \mathbb{C}^{3}$. En esta carta, la foliación es generada por el campo vectorial holomorfo $Z=\sum_{i=1}^{3} Z_{i} \frac{\partial}{\partial z_{i}}$. Si $m_{0}(Z)=\nu$, por el teorema de caracterización de campos con singularidades dicríticas aisladas (ver [1]), existe un polinomio homogéneo 
$P_{\nu-1}$ de grado $\nu-1$, tal que:

$$
Z_{i}\left(z_{1}, z_{2}, z_{3}\right)=z_{i} P_{\nu-1}\left(z_{1}, z_{2}, z_{3}\right)+\sum_{k \geq \nu+1} Z_{i}^{k}\left(z_{1}, z_{2}, z_{3}\right), \quad 1 \leq i \leq 3
$$

Sea $(x, t, s)$ la carta de $\tilde{\mathbb{C}}^{3}$ en la cual el blow-up $E$ centrado en el $0 \in \mathbb{C}^{3}$ es expresado como:

$$
E(x, t, s)=(x, x t, x s)=\left(z_{1}, z_{2}, z_{3}\right) .
$$

En estas coordenadas, las componentes del campo $\tilde{Z}=\tilde{Z}_{1} \frac{\partial}{\partial x}+{ }^{\prime}$ $\tilde{Z}_{2} \frac{\partial}{\partial t}+\tilde{Z}_{3} \frac{\partial}{\partial s}$ que genera a la foliación transformada estricta, se expresan como:

$$
\mid \begin{aligned}
& \tilde{Z}_{1}(x, t, s)=P_{\nu-1}(1, t, s)+\sum_{k \geq \nu+1} x^{k-\nu} Z_{k}^{1}(1, t, s) \\
& \tilde{Z}_{2}(x, t, s)=\sum_{k \geq \nu+1} x^{k-\nu-1}\left[Z_{k}^{2}(x, t, s)-t Z_{k}^{1}(1, t, s)\right] \\
& \tilde{Z}_{3}(x, t, s)=\sum_{k \geq \nu+1} x^{k-\nu-1}\left[Z_{k}^{3}(x, t, s)-s Z_{k}^{1}(1, t, s)\right]
\end{aligned}
$$

Las componentes de $\tilde{Z}$ tienen expresiones análogas en las otras dos cartas $(u, y, v)$ y $(r, w, z)$ de $\tilde{\mathbb{C}}^{3}$, en las cuales $E$ se escribe:

$$
\mid \begin{aligned}
& E(u, y, v)=(u y, y, v y)=\left(z_{1}, z_{2}, z_{3}\right) \\
& E(r, w, z)=(r z, w z, z)=\left(z_{1}, z_{2}, z_{3}\right)
\end{aligned}
$$

Observe que las componentes de $\tilde{Z}$ también pueden sèr expresadas en términos de los transformados estrictos de las componentes de $Z$. En efecto, por ejemplo en la carta $(x, t, s)$, un fácil cálculo nos muestra que:

$$
\tilde{Z}=\tilde{Z}_{1} \frac{\partial}{\partial x}+\frac{\tilde{Z}_{2}-t \tilde{Z}_{1}}{x} \frac{\partial}{\partial t}+\frac{\tilde{Z}_{3}-s \tilde{Z}_{1}}{x} \frac{\partial}{\partial s}
$$

Con las definiciones y notaciones anteriores, podemos demostrar nuestro resultado principal:

Teorema 2. Sea $Z$ un campo vectorial holomorfo con singularidad aislada en $0 \in \mathbb{C}^{3}$, tal que $\tilde{Z}$ tiene singularidades aisladas en el divisor. Si $0 \in \mathbb{C}^{3}$ es una singularidad dicrítica de $Z$ y $m_{\theta}(Z)=\nu$, entonces

$$
\mu_{0}(Z)=\nu^{3}+2 \nu^{2}-2+\sum_{q \in E^{-1}(0)} \mu_{q}(Z)
$$

Prueba. Desde que $\tilde{Z}$ tiene singularidades aisladas, sin pérdida de generalidad, podemos asumir que las siguientes condiciones son satisfechas: 
1. La carta $(x, t, s)$ de $\tilde{\mathbb{C}}^{3}$ contiene todas las singularidades de $\tilde{Z}$.

2. $Z_{\nu+1}^{1}(0,0,1) \neq 0$ y $P_{\nu-1}(0,1,0) \neq 0$.

3. $P_{\nu-1}\left(0, z_{2}, z_{3}\right)$ y $Z_{\nu+1}^{1}\left(0, z_{2}, z_{3}\right)$ son polinomios coprimos.

Usando las propiedades del número de Milnor y del índice de intersección, tenemos:

$$
\begin{aligned}
\sum_{q \in E^{-1}(0)} \mu_{q}(\tilde{Z})= & \\
= & \sum_{q \in E^{-1}(0)} i_{q}\left(\tilde{Z}_{1}, \frac{\tilde{Z}_{2}-t \tilde{Z}_{1}}{x}, \frac{\tilde{Z}_{3}-s \tilde{Z}_{1}}{x}\right) \\
= & \sum_{q \in E^{-1}(0)} i_{q}\left(\tilde{Z}_{1}, \tilde{Z}_{2}, \frac{\tilde{Z}_{3}-s \tilde{Z}_{1}}{x}\right)- \\
& -\sum_{q \in E^{-1}(0)} i_{q}\left(\tilde{Z}_{1}, \dot{x}, \frac{\tilde{Z}_{3}-s \tilde{Z}_{1}}{x}\right)
\end{aligned}
$$

donde (3.1) es válida siempre que la sumatoria:

$\sum_{q \in E^{-1}(0)} i_{q}\left(\tilde{Z}_{1}, x, \frac{\tilde{Z}_{3}-s \tilde{Z}_{1}}{x}\right)$ sea finita. Con el objetivo de probar esta finitud, observamos que:

$$
\begin{aligned}
\sum_{q \in E^{-1}(0)} i_{q}\left(\tilde{Z}_{1}, x, \frac{\tilde{Z}_{3}-s \tilde{Z}_{1}}{x}\right)= & \sum_{p \in \mathbb{C}^{2}} i_{p}\left(P_{\nu-1}(1, t, s), Z_{\nu+1}^{3}(x, t, s)\right)- \\
& -\left(s Z_{\nu+1}^{1}(1, t, s)\right)
\end{aligned}
$$

Sea $H_{\nu+2}$ el polinomio homogéneo de grado $\nu+2$ definido por:

$$
H_{\nu+2}\left(z_{1}, z_{2}, z_{3}\right)=z_{1} Z_{\nu+1}^{3}\left(z_{1}, z_{2}, z_{3}\right)-z_{3} Z_{\nu+1}^{1}\left(z_{1}, z_{2}, z_{3}\right)
$$

la condición 3 implica que $H_{\nu+2}$ y $P_{\nu-1}$ no tienen factores comunes, luego por el Teorema de Bezout:

$$
\sum_{p \in \mathbb{C}^{2}} i_{p}\left(P_{\nu-1}, H_{\nu+2}\right)=(\nu-1)(\nu+2)
$$

De las condiciones 2 y 3 , tenemos que los polinomios $P_{\nu-1}(0,1, v)$ y $H_{\nu+2}(0,1, v)=-v Z_{\nu+1}^{1}(0,1, v)$ no tienen raíces comunes, además $H_{\nu+2}(0,0,1)=-Z_{\nu+1}^{1}(0,0,1) \neq 0$. Por lo tanto, las curvas algebraicas $\left(P_{\nu-1}=0\right)$ y $\left(H_{\nu+2}=0\right)$ no tienen puntos de intersección en la recta del infinito $L_{\infty}=\mathbb{C} P(2)-\mathbb{C}^{2}$, esto significa que el plano afín $\mathbb{C}^{2}$ contiene a todos los puntos de intersección de estas curvas. Luego: 


$$
\begin{aligned}
\sum_{q \in E^{-1}(\theta)} i_{q}\left(\tilde{Z}_{1}, x, \frac{\tilde{Z}_{3}-s \tilde{Z}_{1}}{x}\right) & =\sum_{p \in \mathbb{C}^{2}} i_{p}\left(P_{\nu-1}(1, t, s), H_{\nu+2}(1, t, s)\right) \\
& =\sum_{p \in \mathbb{C}^{2}} i_{p}\left(P_{\nu-1}, H_{\nu+2}\right)=(\nu-1)(\nu+2)
\end{aligned}
$$

Por lo tanto se verifica la igualdad (3.1) y tenemos

$$
\begin{aligned}
\sum_{q \in E^{-1}(0)} i_{q}\left(\tilde{Z}_{1}, \tilde{Z}_{2}, \frac{\tilde{Z}_{3}-s \tilde{Z}_{1}}{x}\right)= & (\nu-1)(\nu+2)+ \\
& +\sum_{q \in E^{-1}(0)} \mu_{q}(\tilde{Z})
\end{aligned}
$$

Calculemos ahora la sumatoria $\sum_{q \in E^{-1}(0)} i_{q}\left(\tilde{Z}_{1}, \tilde{Z}_{2}, \frac{\tilde{Z}_{3}-s \tilde{Z}_{1}}{x}\right)$. Sea $H$ la función analítica:

$$
\begin{aligned}
H\left(z_{1}, z_{2}, z_{3}\right) & =z_{1} Z_{3}\left(z_{1}, z_{2}, z_{3}\right)-z_{3} Z_{1}\left(z_{1}, z_{2}, z_{3}\right) \\
& =\sum_{k \geq \nu+1}\left(z_{1} Z_{k}^{3}\left(z_{1}, z_{2}, z_{3}\right)-z_{3} Z_{k}^{1}\left(z_{1}, z_{2}, z_{3}\right)\right)
\end{aligned}
$$

De la condición 3 , se sigue fácilmente que $0 \in \mathbb{C}^{3}$ es un punto de intersección aislado de las superficies analíticas $\left(Z_{1}=0\right),\left(Z_{2}=0\right)$ y $(H=0)$. Por propiedades del índice de intersección, tenemos:

$$
\begin{aligned}
i_{0}\left(Z_{1}, Z_{2}, H\right) & =i_{0}\left(Z_{1}, Z_{2}, z_{1} Z_{3}\right) \\
& =i_{0}\left(Z_{1}, Z_{2}, z_{1}\right)+i_{0}\left(Z_{1}, Z_{2}, Z_{3}\right) \\
& =i_{0}\left(Z_{1}, Z_{2}, z_{1}\right)+\mu_{0}(Z)
\end{aligned}
$$

Del Teorema 1, tenemos:

$$
\begin{aligned}
i_{0}\left(Z_{1}, Z_{2}, z_{1}\right) & =i_{0}\left(\sum_{k \geq \nu+1} Z_{k}^{1}, Z_{2}, z_{1}\right) \\
& =\nu(\nu+1)+\sum_{q \in E^{-1}(0)} i_{q}\left(\tilde{Z}^{\prime}, \tilde{Z}_{2}, \tilde{\pi}_{1}\right)
\end{aligned}
$$

en donde $\tilde{Z}^{\prime}=\sum_{k \geq \nu+1} Z_{k}^{1}$ y $\pi_{1}\left(z_{1}, z_{2}, z_{3}\right)=z_{1}$. Desde que $\tilde{\pi}_{1}\left(z_{1}, z_{2}, z_{3}\right)=$ 1, las superficies $\left(\tilde{Z}^{\prime}=0\right),\left(\tilde{Z}_{2}=0\right)$ y $\left(\tilde{\pi}_{1}=0\right)$ no tienen puntos de intersección en la carta $(x, t, s)$. en las otras dos eartas: $(u, y, w)$ y $(r, w, z)$, 
los puntos de intersección de estas superficies que se encuentran en el divisor, vienen dados por las soluciones de los sistemas:

$$
\mid \begin{aligned}
& \tilde{Z}^{\prime}(u, 0, v)=Z_{\nu+1}(u, 1, v)=0 \\
& \tilde{Z}_{2}(u, 0, v)=P_{\nu-1}(u, 1, v)=0 \\
& \tilde{\pi}_{1}(u, 0, v)=u=0 \\
& \tilde{Z}^{\prime}(r, w, 0)=Z_{\nu+1}(r, w, 1)=0 \\
& \tilde{Z}_{2}(r, w, 0)=w P_{\nu-1}(r, w, 1)=0 \\
& \tilde{\pi}_{1}(r, w, 0)=r=0
\end{aligned}
$$

pero de las condiciones 2 y 3 , deducimos que los dos sistemas anteriores no tienen solución, luego (6.1) implica que

$$
i_{0}\left(Z_{1}, Z_{2}, z_{1}\right)=\nu(\nu+1)
$$

Usando nuevamente el Teorema 1, tenemos:

$$
i_{0}\left(Z_{1}, Z_{2}, H\right)=\nu^{2}(\nu+2)+\sum_{q \in E^{-1}(0)} i_{q}\left(\tilde{Z}_{1}, \tilde{Z}_{2}, \tilde{H}\right)
$$

siempre que las superficies $\left(\tilde{Z}_{1}=0\right),\left(\tilde{Z}_{2}=0\right)$ y $(\tilde{H}=0)$ se intersecten en puntos aislados del divisor. En la carta $(x, t, s)$ esto se cumple (ver (3.2)) y en las otras dos cartas $(u, y, v)$ y $(r, w, z)$ estos puntos de intersección (los cuales no pertenecen a la carta $(x, t, s)$ ), son soluciones de los sistemas:

$$
\begin{aligned}
& \mid \begin{array}{l}
\tilde{Z}_{1}(0,0, v)=0 \\
\tilde{Z}_{2}(0,0, v)=P_{\nu-1}(0,1, v)=0 \\
\tilde{H}(0,0, v)=-v Z_{\nu+1}(0,1, v)=0
\end{array} \\
& \mid \begin{array}{l}
\tilde{Z}_{1}(0, w, 0)=0 \\
\tilde{Z}_{2}(0, w, 0)=w P_{\nu-1}(0, w, 1)=0 \\
\tilde{H}(0,0, v)=-Z_{\nu+1}(0, w, 1)=0
\end{array}
\end{aligned}
$$

Nuevamente, por las condiciones 2 y 3, los sistemas anteriores no admiten solución. Se sigue de (6.3) que:

$(6.7) i_{0}\left(Z_{1}, Z_{2}, H\right)=\nu^{2}(\nu+2)+\sum_{q \in E^{-1}(0)} i_{q}\left(\tilde{Z}_{1}, \tilde{Z}_{2}, \frac{\tilde{Z}_{3}-s \tilde{Z}_{1}}{x}\right)$

Finalmente, de (3), (7), (5) y (2), tenemos: 


$$
\begin{aligned}
\mu_{0}(Z) & =i_{0}\left(Z_{1}, Z_{2}, H\right)-i_{0}\left(Z_{1}, Z_{2}, z_{1}\right) \\
& =\nu^{2}(\nu+2)+\sum_{q \in E^{-1}(0)} i_{q}\left(\tilde{Z}_{1}, \tilde{Z}_{2}, \frac{\tilde{Z}_{3}-s \tilde{Z}_{1}}{x}\right)-\nu(\nu+1) \\
& =\nu^{2}(\nu+2)-\nu(\nu+1)+(\nu-1)(\nu+2)+\sum_{q \in E^{-1}(0)} \mu_{q}(\tilde{Z}) \\
& =\nu^{3}+2 \nu^{2}-2+\sum_{q \in E^{-1}(0)} \mu_{q}(\tilde{Z})
\end{aligned}
$$

lo cual prueba el Teorema 2.

\section{Observaciones:}

1. La técnica usada para la demostración del Teorema 2, es una extensión a dimensión 3 de la utilizada en [3] para probar el resultado análogo para singularidades dicríticas aisladas en $\mathbb{C}^{2}$.

2. La generalización del Teorema 2 a cualquier dimensión $n$ es probada en [2]. En esta referencia, usando métodos topológicos, se demuestra que si $Z$ es un campo vectorial holomorfo con una singularidad dicrítica aislada en $0 \in \mathbb{C}^{n}$ tal que el transformado estricto $\tilde{Z}$ no tiene singularidades en el divisor entonces

$$
\mu_{0}(Z)=\sum_{k=0}^{n} n() k \frac{n+1-2 k}{n+1-k} \nu^{n-k}+\sum_{q \in E^{-1}(0)} \mu_{q}(\tilde{Z}) .
$$

en donde $m_{0}(Z)=\nu$. Una demostración algebraica de este resultado para $n \geq 4$ aún no se ha encontrado.

\section{BIBLIOGRAFÍA}

[1] R. Benazic, Caracterización de singularidades dicríticas en foliaciones de dimension uno, PESQUIMAT, Vol. 1, N 1, (1998), p. 73-81.

[2] R. Benazic, A resolution theorem for absolutely isolated singularities of holomorphic vector fields, Bol. Soc. Bras. Mat., Vol 28, N 1, (1997), p. 211-231.

[3] C. Camacho, Holomorphic Dynamical Systems, Summer School on Dynamical Systems, ICTP, Trieste - Italia, (16 August - 9 September, 1988).

[4] C. Camacho, F. Cano, P. Sad, Absolutely isolated singularities of holomorphic vector fields, Invent. math. 98, (1989), p. 351-369.

[5] D. Cerveau, J. Mattei, Formes Intégrables Holomorphes Singulières, Asterisque, $97,(1982)$.

[6] E. Chirka, Complex Analytic Sets, MIA, Kluwer Academic Publishers. Dordrecht, Boston, London (1989).

[7] W. Fulton, Intersection Theory, Berlin, Heidelberg, New York: Springer (1984). 
[8] P. Griffiths, J. Harris, Principles of Algebraic Geometry, Wiley-Intersciencie, New York, (1978).

[9]. R. Gunning, H. Rossi, Analytic functions of several complex variables, PrenticeHall, Engleweod Cliffs, NJ., (1965).

[10] J. Mattei, R. Moussu, Holonomie et intégrales premières, Ann. Sci. Ecole. Norm. Sup. (4) 13 (1980) p. 469-523.

[11] H. Whitney, Complex Analytic Varieties, Addison-Wesley Publishing Company, (1972). 